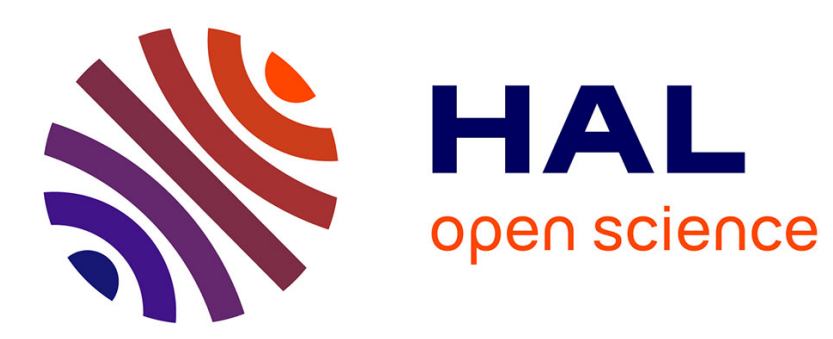

\title{
A Doubling Construction for 3-Uniform Friendship Hypergraphs with the Universal Pairs Property
}

\author{
Nathann Cohen
}

\section{To cite this version:}

Nathann Cohen. A Doubling Construction for 3-Uniform Friendship Hypergraphs with the Universal Pairs Property. Journal of Combinatorial Designs, 2016, 24 (7), pp.336-339. 10.1002/jcd.21509 . hal-01316029

\section{HAL Id: hal-01316029 \\ https://hal.science/hal-01316029}

Submitted on 14 May 2016

HAL is a multi-disciplinary open access archive for the deposit and dissemination of scientific research documents, whether they are published or not. The documents may come from teaching and research institutions in France or abroad, or from public or private research centers.
L'archive ouverte pluridisciplinaire HAL, est destinée au dépôt et à la diffusion de documents scientifiques de niveau recherche, publiés ou non, émanant des établissements d'enseignement et de recherche français ou étrangers, des laboratoires publics ou privés. 


\title{
A doubling construction for 3-uniform friendship hypergraphs with the Universal Pairs Property
}

\author{
Nathann Cohen*
}

\begin{abstract}
In this note, we present a simple doubling construction for 3-uniform friendship hypergraphs which generalizes the cubeconstructed hypergraphs from Jørgensen and Sillasen [3]. As a byproduct, we build point-transitive 3 -uniform friendship hypergraphs of sizes $20.2^{k}$ and $28.2^{k}$ for all $k \geq 0$.
\end{abstract}

\section{Introduction}

The Friendship Theorem, proved in 1966 by Erdős, Rényi, and Sós [1], asserts that the only graphs in which any two vertices have exactly one common neighbour are those that can be obtained from a perfect matching by the addition of a universal vertex.

In 1976, Sós [4] generalized this property to 3-uniform hypergraphs (see below). In the following, we will define a hypergraph $\mathcal{H}$ on a ground set $X$ as a subset of $2^{X}$ (i.e. the powerset of $X$ ). We will often write $X=V(\mathcal{H})$. A hypergraph $\mathcal{H}$ is said to be 3 -uniform if all its sets have cardinality 3 . We also call degree of a point $x \in V(\mathcal{H})$ the number of sets from $\mathcal{H}$ containing it.

Definition 1 (Sós 1976). A 3-uniform hypergraph $\mathcal{H}$ has the friendship property if for any three distinct points $x_{1}, x_{2}, x_{3} \in V(\mathcal{H})$ there is a unique $y \in V(\mathcal{H})$ such that $\left\{x_{1}, x_{2}, y\right\},\left\{x_{2}, x_{3}, y\right\}$ and $\left\{x_{1}, x_{3}, y\right\}$ belong to $\mathcal{H}$.

In the same paper, it was proved that a 3-uniform friendship hypergraph on $n$ points exists whenever $n \equiv 2,4(\bmod 6)$. Indeed, similarly to the construction of friendship graphs, a hypergraph obtained from Steiner Triple System $\mathcal{S}$ on $n-1$ points by the addition of a universal point $v$ (i.e. a point of degree $\left.\left(\begin{array}{c}n-1 \\ 2\end{array}\right)\right)$ has the desired property.

Contrarily to the graph setting, however, it does not hold that all 3-uniform hypergraph contain a universal point [2]. In 2014, Jørgensen and Sillasen [3] generalized the known examples of sizes 8,16 and 32 into a family of cubeconstructed hypergraphs and obtained the first infinite family of 3-uniform friendship hypergraphs without universal points.

${ }^{*}$ CNRS - Laboratoire de Recherche en Informatique, Bâtiment 650, Université Paris Sud XI, 91405 Orsay, France - ncohen@lri.fr 
Theorem 2 (Jørgensen and Sillasen 2014). For any $k>2$ there exists a 3uniform friendship hypergraph on $2^{k}$ points containing no universal point.

Those cubeconstructed hypergraphs are defined from the distance matrix of the hypercube graph on $2^{k}$ points. They are also point-transitive, i.e. the automorphism group of a cubeconstructed hypergraph acts transitively on its ground set. This led them to Conjecture 3 (note that a point-transitive 3uniform friendship hypergraph on $>4$ points cannot contain a universal point).

Conjecture 3 (Jørgensen and Sillasen 2014). For any $n$ divisible by 4 and not divisible by 3 there exists a point-transitive 3-uniform friendship hypergraph on $n$ points.

In this note, we present a simple doubling construction for 3-uniform friendship hypergraphs satisfying an additional property.

Definition 4. Two distinct points $x, y \in V(\mathcal{H})$ are said to form a universal pair if $\{x, y, z\} \in \mathcal{H}$ for any $z \in V(\mathcal{H})$ distinct from $x, y$. A hypergraph $\mathcal{H}$ is said to have the universal pairs property if its ground set can be partitionned into universal pairs.

As this property is preserved through the doubling construction, any instance of such a hypergraph yields an infinite family.

\section{A doubling construction}

Construction 5. If there exists a 3-uniform frienship hypergraph $\mathcal{H}$ of size $n$ with the universal pairs property, then there exists a 3-uniform frienship hypergraph $\mathcal{H}^{\prime}$ of size $2 n$ with the universal pairs property. Furthermore, both point-transitivity and the abscence of universal point are preserved.

Proof. Let $P$ be a partition of $V(\mathcal{H})$ into universal pairs, and let $x \mapsto \bar{x}$ associate to each point $x \in V(\mathcal{H})$ the point $\bar{x}$ with which it is matched in $P$. We build $\mathcal{H}^{\prime}$ defined on $V\left(\mathcal{H}^{\prime}\right)=V(\mathcal{H}) \times\{0,1\}$ with the following set of triples:

- $\{x 0, y 0, z 1\}$ and $\{x 1, y 1, z 0\}$, for $\{x, y, z\} \in \mathcal{H}$

$(6|\mathcal{H}|$ triples $)$

- $\{x 0, x 1, \bar{x} 0\}$ and $\{x 0, x 1, \bar{x} 1\}$, for $x \in V(\mathcal{H})$

(2n triples)

Note that no triple is contained in $V(\mathcal{H}) \times\{0\}$ or $V(\mathcal{H}) \times\{1\}$. Note also that $\{x 0, \bar{x} 1\}$ is a universal pair of $\mathcal{H}^{\prime}$ for every $x \in V(\mathcal{H})$, and that any automorphism $\tau$ of $\mathcal{H}$ yields an automorphism of $\mathcal{H}^{\prime}$ with $\tau^{\prime}: x b \mapsto \tau(x) b$. Furthermore, the automorphism $r: x 0 \mapsto x 1, x 1 \mapsto x 0$ of $\mathcal{H}^{\prime}$ ensures that $\mathcal{H}^{\prime}$ is point-transitive when $\mathcal{H}$ is point-transitive. Let us now check that $\mathcal{H}^{\prime}$ is a 3 -uniform friendship hypergraph, splitting cases for each possible triple:

- $\{x 0, y 0, z 0\}$ - No point $w 0$ can be an extension of $\{x 0, y 0, z 0\}$. Additionally, $x 1$ cannot be an extension of $\{x 0, y 0, z 0\}$ as the only two triples of $\mathcal{H}^{\prime}$ that contain $\{x 1, x 0\}$ are $\{x 1, x 0, \bar{x} 1\}$ and $\{x 1, x 0, \bar{x} 0\}$, one of which 
contains two elements from $V(\mathcal{H}) \times\{1\}$ (similarly, $y 1$ and $z 1$ are not possible extensions). Consequently, $w 1$ is an extension of $\{x 0, y 0, z 0\}$ in $\mathcal{H}^{\prime}$ if and only if $w$ is an extension of $\{x, y, z\}$ in $\mathcal{H}$.

- $\{x 0, y 0, z 1\}$ with $x, y, z$ distinct - No point $w 0$ can be an extension of $\{x 0, y 0, z 1\}$. Additionally, $x 1$ cannot be an extension of $\{x 0, y 0, z 1\}$ as the only two triples of $\mathcal{H}^{\prime}$ that contain $\{x 1, x 0\}$ are $\{x 1, x 0, \bar{x} 1\}$ and $\{x 1, x 0, \bar{x} 0\}$ and $\{y 0, z 1\} \neq\{\bar{x} 0, \bar{x} 1\}$ (similarly, $y 1$ is not a possible extensions). Consequently, $w 1$ is an extension of $\{x 0, y 0, z 0\}$ in $\mathcal{H}^{\prime}$ if and only if $w$ is an extension of $\{x, y, z\}$ in $\mathcal{H}$.

- $\{x 0, y 0, x 1\}-$ No point $w 0$ can be an extension of $\{x 0, y 0, x 1\}$, and the only point $w$ such that $\{x 0, x 1, w 1\} \in \mathcal{H}^{\prime}$ is $w=\bar{x}$. Consequently, the only possible extension of the triple is $\bar{x} 1$.

$y \neq \bar{x}$ ) in this case $x \bar{x}$ is a universal pair of $\mathcal{H}$ and in particular $x \bar{x} y \in \mathcal{H}$, which implies $\{y 0, x 1, \bar{x} 1\} \in \mathcal{H}$. As $\{x 0, \bar{x} 1\}$ is a universal pair of $\mathcal{H}^{\prime}$ we also have $\{x 0, \bar{x} 1, y 0\},\{x 0, \bar{x} 1, x 1\} \in \mathcal{H}^{\prime}$ and thus $\bar{x} 1$ is an extension of $\{x 0, y 0, x 1\}$.

$y=\bar{x})$ in this case the three triples needed are $\{x 0, x 1, \bar{x} 1\},\{x 0, \bar{x} 0, \bar{x} 1\}$ and $\{\bar{x} 0, x 1, \bar{x} 1\}$ which all belong to $\mathcal{H}^{\prime}$.

By symmetry with respect to the automorphism $r$, this exhausts all cases.

This construction has the following corollary:

Corollary 6. If there exists a 3-uniform friendship hypergraph of size $n$ with the universal pairs property (and no universal point), there exists a 3-uniform friendship hypergraph (with no universal point) of size $2^{k} n$ for every $k \in \mathbb{N}$.

Applied to the trivial 3-uniform friendship hypergraph of size 4, we obtain the infinite family first presented in [3]. In the same paper, Jørgensen and Sillasen proved the existence of two instances of sizes $n=20$ and $n=28$ through computer search. We quote one such instance for each value of $n$. Note that each set of size 4 is to be replaced by the four sets of size 3 contained in it.

$$
\begin{gathered}
n=20-\text { generated by the additive action of } \mathbb{Z} / 20 \mathbb{Z} \text { on: } \\
\{i, j, i+10, j+10\} \text { for all } i, j \in\{0, \ldots, 9\} \text { and } \\
\{0,1,3,14\},\{0,1,9,15\},\{0,2,4,7\} \\
n=28-\text { generated by the additive action of } \mathbb{Z} / 28 \mathbb{Z} \text { on: } \\
\{i, j, i+14, j+14\} \text { for all } i, j \in\{0, \ldots, 13\} \text { and } \\
\{0,1,4,17\},\{0,1,5,20\},\{0,1,7,13\},\{0,2,4,23\},\{0,3,8,19\},\{0,3,10,20\}
\end{gathered}
$$

Those two point-transitive hypergraphs have the universal pairs property, as is shown by the two partitions $\{\{i, i+10\}: i \in[20]\}$ and $\{\{i, i+14\}: i \in[28]\}$. Consequently:

Corollary 7. There exist a point-transitive 3-uniform friendship hypergraphs (thus without universal point) of size $20.2^{k}$ and $28.2^{k}$ for every $k \geq 0$. 


\section{Conclusion}

This doubling construction can be seen as a step toward Conjecture 3, for it reduces it to the construction of 3-uniform friendship hypergraphs of size $4 n$ (for $n$ odd and not divisible by 3 ) with the universal pairs property. It seems, however, quite difficult to reach as humble a goal as build an instance of size 44 from the very rich structure of the known instances of sizes 20 and 28. Let it be remarked as an evidence that the sets $\{0,1,3,14\},\{0,1,9,15\},\{0,2,4,7\}$ (involved in the $n=20$ instance), seen as subsets of $\mathbb{Z} / 10 \mathbb{Z}$, are actually cyclic generators of the unique Steiner Quadruple System on 10 points.

\section{References}

[1] P Erdős, A Rényi, and VT Sós. On a problem of graph theory. Studia Sci. Math. Hungar, 1:215-235, 1966.

[2] S. Hartke and J. Vandenbussche. On a question of Sós about 3-uniform friendship hypergraphs. Journal of Combinatorial Designs, 16(3):253-261, 2008 .

[3] L Jørgensen and A. Sillasen. On the existence of friendship hypergraphs. Journal of Combinatorial Designs, 2014.

[4] VT Sós. Some remarks on the connection of graph theory, finite geometry and block designs. Colloquio Internazionale sulle Teorie Combinatorie, 2:223-233, 1976. 